\title{
Characterization of a cryptic KMT2A/AFF1 gene fusion by mate-pair sequencing (MPseq) in a young adult with newly diagnosed B-lymphoblastic leukemia
}

\author{
Jess F. Peterson ${ }^{1}$ - Stephanie A. Smoley ${ }^{1}$ - Ivy M. Luoma ${ }^{1} \cdot$ Beth A. Pitel $^{1} \cdot$ Christopher S. Rice $^{1}$. \\ Jonna C. Benevides Demasi ${ }^{1}$. George Vasmatzis ${ }^{2}$ • James B. Smadbeck ${ }^{2}$. Tong Yang ${ }^{3}$ • Patricia T. Greipp ${ }^{1}$. \\ Rhett P. Ketterling ${ }^{1} \cdot$ Linda B. Baughn ${ }^{1}$
}

Received: 6 March 2019 / Accepted: 22 April 2019 / Published online: 3 May 2019

(C) Springer-Verlag GmbH Germany, part of Springer Nature 2019

\begin{abstract}
The detection of recurrent chromosomal rearrangements in B-lymphoblastic leukemia/lymphoma (B-ALL/LBL) is critical for patient management decisions. We present a newly diagnosed case of B-ALL in a young adult with a cryptic $K M T 2 A / A F F 1$ fusion that was unappreciable by conventional chromosome and fluorescence in situ hybridization (FISH) KMT2A break-apart probe studies. To further characterize this abnormality, a next-generation sequencing strategy, mate-pair sequencing (MPseq) was performed and characterized a cryptic, insertional rearrangement that created $K M T 2 A / A F F 1$ gene fusion. This case highlights the superior precision and resolution capabilities of NGS when compared to traditional cytogenetic methodologies, including conventional chromosome and FISH studies.
\end{abstract}

Keywords $K M T 2 A(M L L) \cdot A F F 1(A F 4) \cdot$ B-lymphoblastic leukemia/lymphoma (B-ALL/LBL) $\cdot$ Next-generation sequencing (NGS) $\cdot$ Mate-pair sequencing (MPseq)

\section{Introduction}

The AFF1 gene (4q21.3-q22.1) (also known as AF4) is the most common KMT2A gene (11q23.3) fusion partner observed in all age groups of B-lymphoblastic leukemia/ lymphoma (B-ALL/LBL) and is associated with an unfavorable prognosis [1-6]. The detection of KMT2A rearrangements in B-ALL/LBL and other acute leukemia subtypes mainly relies upon conventional chromosome and/or

Jess F. Peterson

peterson.jess@mayo.edu

1 Division of Laboratory Genetics and Genomics, Department of Laboratory Medicine and Pathology, Mayo Clinic, 200 First Street SW, Rochester, MN 55905, USA

2 Center for Individualized Medicine-Biomarker Discovery, Mayo Clinic, Rochester, MN, USA

3 Department of Pathology and Laboratory Medicine, Ochsner Medical Center, New Orleans, LA, USA fluorescence in situ hybridization (FISH) studies, including $K M T 2 A$ break-apart probes (BAP) and dual-color, dualfusion (D-FISH) probe sets that can detect $K M T 2 A$ rearrangements or specific $K M T 2 A$ gene fusion partners, respectively. Based on the experience from our genomics laboratory, the most commonly reported KMT2A gene partners ( $A F F 1$, $A F D N, M L L T 3, E L L, M L L T 1)$, with the exception of MLLT10, usually create balanced rearrangements as indicated by conventional chromosome and/or D-FISH studies [7, 8].

Herein, we present a 25 -year-old female with newly diagnosed B-ALL with normal conventional chromosome and KMT2A BAP studies. However, chromosomal microarray analysis (CMA) revealed an $\sim 584 \mathrm{~kb}$ heterozygous deletion that spanned exons $1-4$ of the $A F F 1$ gene region, suggesting a potential rearrangement that was subsequently confirmed as "cryptic" KMT2A/AFF1 fusion by our KMT2A/AFF1 D-FISH probe set. To further characterize this cryptic rearrangement, we utilized mate-pair sequencing (MPseq), a next-generation sequencing (NGS) strategy that can resolve structural abnormalities with greater resolution and precision compared to conventional chromosome and FISH methodologies [9-11]. 


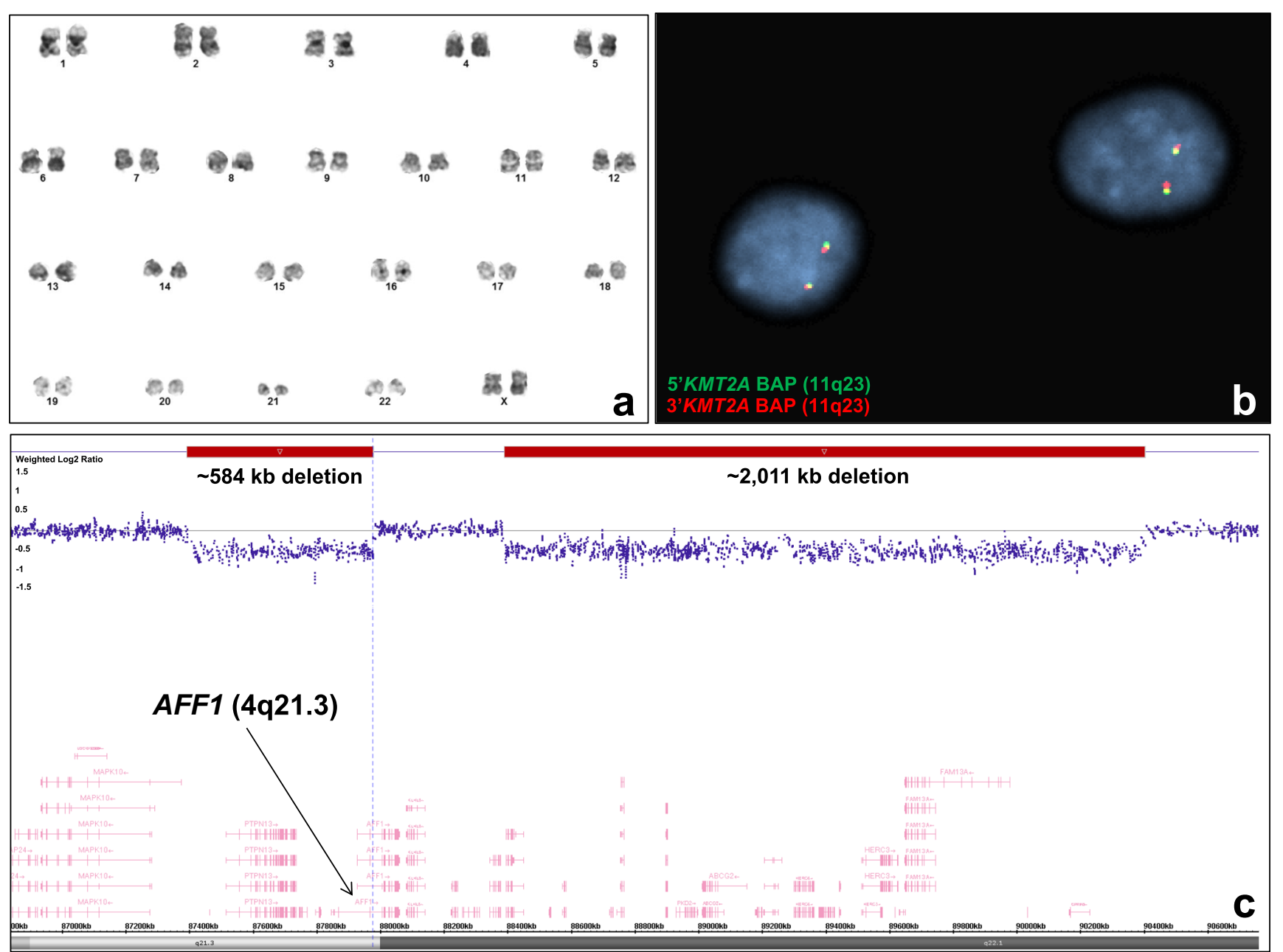

Fig. 1 a Representative normal female karoygram $(46, \mathrm{XX})$. No structural or numerical abnormalities were observed in 20 metaphases. b Representative interphase nuclei demonstrating a normal result of two intact KMT2A fusion signals (yellow) using the KMT2A FISH BAP set. This signal pattern is observed when the KMT2A gene regions are not disrupted (negative). c Microarray analysis demonstrating an $\sim 584 \mathrm{~kb}$ heterozygous deletion (indicated by the left horizontal red rectangle) that spans the 5'AFF1 gene region at 4q21.3 (vertical dashed blue line). This finding indicated a potential $A F F 1$ gene rearrangement. In addition, an $2011 \mathrm{~kb}$ heterozygous deletion was observed telemetric to the $\sim 584$ deletion that was separated by an $\sim 409 \mathrm{~kb}$ gap with a normal copy number

and immunophenotypic findings were consistent with a diagnosis of B-ALL/LBL.

\section{Materials and methods}

\section{Conventional chromosome and fluorescence in situ hybridization (FISH) studies}

All genomic studies were performed on the diagnostic bone marrow aspirate specimen. For conventional chromosome studies, G-banding by trypsin using Leishman stain was performed on bone marrow cells that were cultured and harvested as per protocol. A total of 20 metaphases were fully analyzed. The bone marrow aspirate specimen was processed for FISH according to 

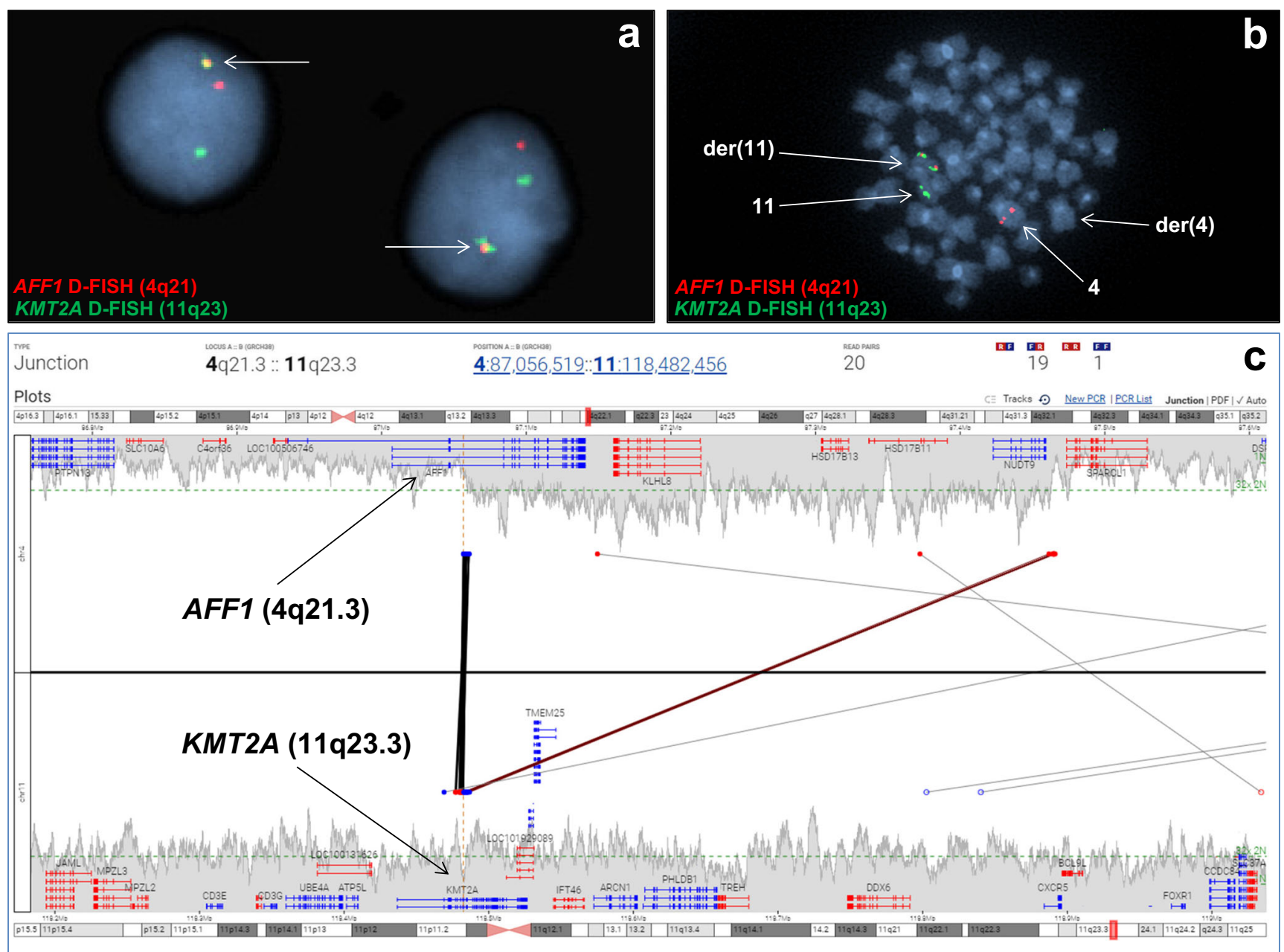

Fig. 2 a Representative interphase nuclei using the $K M T 2 A / A F F 1$ DFISH probe set demonstrating a single yellow fusion signal (arrow) indicating KMT2A/AFF2 fusion. This signal pattern was observed in 480 of $500(96 \%)$ interphase nuclei. b Representative sequential FISH result performed on a "normal" metaphase using the KMT2A/AFF1 D-FISH

specimen-specific laboratory protocols and subjected to standard pretreatment, hybridization, and fluorescence microscopy. A commercially available KMT2A BAP set (Abbott Molecular, Des Plaines, IL) and "laboratory developed" KMT2A/AFF1 D-FISH probes set were utilized. Conventional chromosome and FISH results were interpreted by a board-certified clinical cytogeneticist (ABMGG).

\section{Mate-pair sequencing (MPseq)}

DNA was processed using Illumina Nextera Mate Pair library kit (Illumina, San Diego, CA), multiplexed at two samples per lane, and sequenced (Rapid Run mod) on the Illumina HiSeq 2500 using 101-basepair reads and paired-end sequencing. Data were aligned to the reference genome (GRCh38) using BIMAv3, and abnormalities were characterized using probe set. A single $K M T 2 A / A F F 1$ fusion signal was detected on the distal long arm of chromosome 11. c Mate-pair sequencing (MPseq) results. Junction plot demonstrating the insertion of a segment from chromosome $4 \mathrm{q}$ into chromosomal region 11q23.3. This insertional event resulted in KMT2A/AFF1 fusion

SVAtools and Ingenium, both in-house developed bioinformatics tools $[9,10]$.

\section{Results}

All genomic studies were performed on a submitted bone marrow aspirate specimen. Twenty metaphases were fully analyzed and indicated an apparently normal female karyotype (46,XX) (Fig. 1a). A comprehensive B-ALL FISH panel, including the KMT2A BAP set (Fig. 1b), was performed and all results were normal.

Based on the normal conventional chromosome and FISH studies, chromosomal microarray analysis was performed to identify potential abnormalities of clinical significance that were unappreciable by traditional cytogenetic methodologies. Microarray analysis revealed an $\sim 584 \mathrm{~kb}$ heterozygous deletion including exons $1-4$ of the $A F F 1$ gene region 


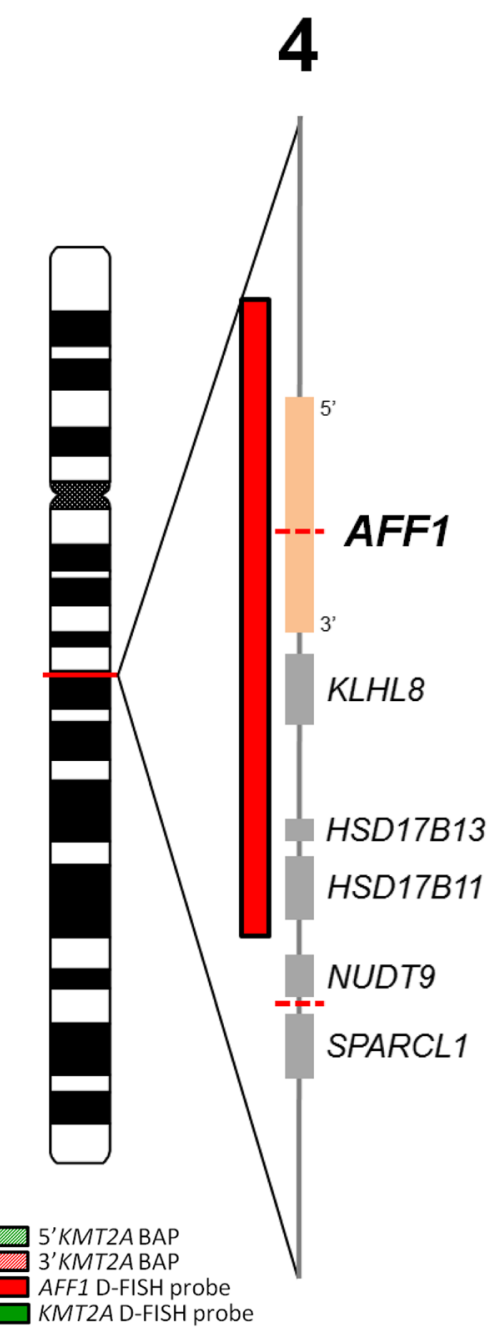

Fig. 3 A focused view of the $A F F 1$ and $K M T 2 A$ gene regions on each normal copy of chromosome 4 and 11 , in addition to the derivative chromosome 11. Horizontal dashed red lines indicate breakpoints. Vertical rectangles with green and red stripes indicate the $5^{\prime} K M T 2 A$ and $3^{\prime} K M T 2 A$ break-apart probe (BAP) footprints, respectively. Solid red and green vertical rectangles indicate the $A F F 1$ and $K M T 2 A$ dual-color, dualfusion (D-FISH) probe footprints, respectively. An $\sim 409 \mathrm{~kb}$ segment of

(NM_001166693) (Fig. 1c). In addition, an $\sim 2011 \mathrm{~kb}$ heterozygous deletion was observed telemetric to the $\sim 584$ deletion separated by an $\sim 409 \mathrm{~kb}$ intact portion of DNA which included a normal copy number for the remaining exons $5-21$ of the $A F F 1$ gene (Fig. 1c). Suspicious for a potential cryptic $K M T 2 A / A F F 1$ fusion, we performed our KMT2A/AFF1 DFISH probe set that is usually reserved for reflex testing following a positive KMT2A BAP study. A single KMT2A/AFF1 fusion signal was detected in 480 of $500(96 \%)$ interphase nuclei (Fig. 2a). Subsequently, serial FISH studies performed on apparently "normal" metaphases demonstrated the single KMT2A/AFF1 fusion signal located on the distal long arm of an apparently normal chromosome 11 (Fig. 2b).

To further characterize the cryptic KMT2A/AFF1 fusion, MPseq was performed and confirmed an $\sim 409 \mathrm{~kb}$ segment
11

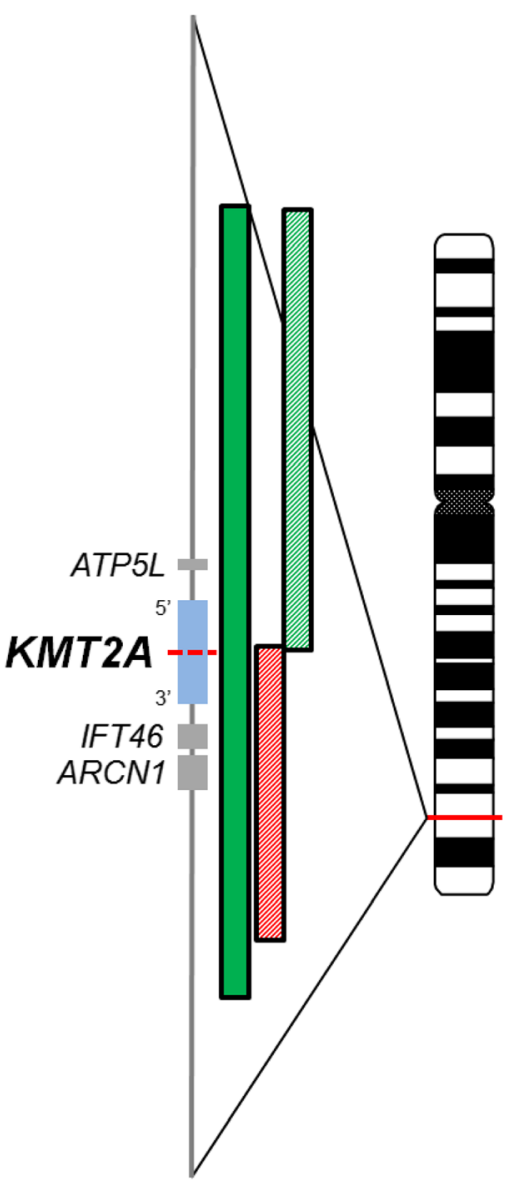

4q21.3-q22.1 was inserted into $11 \mathrm{q} 23.3$, resulting in a $5^{\prime} K M T 2 A$ (exons 1-8, NM_005933) and 3'AFF1 (exons 5-21, NM_001116693) gene fusion. The $\sim 409 \mathrm{~kb}$ insertion is not sufficient to disrupt the KMT2A BAP probe, thus resulting in a false-negative result. However, the juxtaposition of the $A F F 1$ and KMT2A D-FISH footprints generates a single fusion signal as observed in $96 \%$ of 500 interphase nuclei

of 4q21.3-q22.1, including exons 5-21 of the $A F F 1$ gene, and was inserted into chromosomal region 11q23.3, resulting in a 5'KMT2A (exons 1-8, NM_005933) and 3'AFF1 (exons 521, NM_001166693) gene fusion (Fig. 2c). Sanger sequencing subsequently confirmed the KMT2A/AFF1 fusion identified by MPseq.

\section{Discussion}

At our institution, microarray analysis is performed to detect unbalanced chromosomal gains and losses of diagnostic and/or prognostic significance in cases of B-ALL/LBL in the absence of a clear primary abnormality as identified by conventional chromosome and FISH studies. Since our 
patient had normal chromosome and FISH studies, including the KMT2A BAP, chromosomal microarray was performed and revealed an $\sim 584 \mathrm{~kb}$ heterozygous deletion that spanned exons 1-4 of the $A F F 1$ gene region. Considering the diagnosis of B-ALL and the known $A F F 1$ gene fusion association with $K M T 2 A$, we pursued additional testing to evaluate for a potential cryptic $K M T 2 A / A F F 1$ fusion. Using our KMT2A/AFF1 D-FISH probe set, we confirmed a KMT2A/AFF1 fusion in 96\% (480 of 500) of interphase cells. This result was also confirmed by metaphase FISH studies revealing that the cryptic KMT2A/AFF1 fusion was located on the distal long arm of chromosome 11. Based on the results collected thus far, we predicted an $\sim 409 \mathrm{~kb}$ insertion of chromosome 4q21.3-q22.1 into the $11 \mathrm{q} 23.3$ chromosomal region that resulted in the KMT2A/AFF1 fusion; however, the precise molecular characterization of this fusion could not be achieved using existing FISH studies. MPseq confirmed an $\sim 409 \mathrm{~kb}$ insertion of 4q21.3-q22.1 into the $11 \mathrm{q} 23.3$ chromosomal region resulting in a $5^{\prime}$ KMT2A (exons 1-8, NM_005933) and 3'AFF1 (exons 521, NM_001166693) gene fusion (Fig. 3). Taken together, CMA identified the initial clue to the KMT2A/AFF1 fusion and MPseq provided the mechanism and explanation for the normal chromosome and KMT2A BAP studies due to the minute $\sim 409 \mathrm{~kb} 4 \mathrm{q}$ insertion that was too small to be appreciated by either methodology.

The detection of recurrent chromosomal rearrangements in B-ALL is critical for prognostic and treatment-related decisions and most genomics laboratories rely on conventional chromosome and/or FISH studies for their detection $[1,3$, 4]. While conventional chromosome analysis enables a low resolution (limit of detection for structural abnormalities: $10 \mathrm{Mb}$ ) genome-wide view of individual neoplastic cells, cryptic or subtle rearrangements are undetectable without ancillary studies, most commonly interrogated by FISH. While targeted to specific genomic regions, FISH studies provide a significantly higher resolution and are capable of detecting balanced, unbalanced, and cryptic rearrangements that are unappreciable by conventional chromosome studies; although, as demonstrated in this case, some insertions may be too subtle for detection using BAP FISH strategies. However, NGS strategies like MPseq have now surpassed the resolution and precision capabilities of FISH as illustrated by this cryptic B-ALL clone. MPseq is a whole-genome sequencing method that has been developed for characterizing structural variants that often go undetected by short-read paired-end sequencing methods. The generation of $2-5 \mathrm{~kb}$ fragments with 101 base pairs sequenced on each fragment end is optimized during library preparation, resulting in a lower read depth necessary to detect structural variants throughout the genome. NGSbased strategies like MPseq are emerging during an opportune time, as chromosomal rearrangements of diagnostic, prognostic, and/or theranostic importance continue to be discovered
$[12,13]$. This new approach to cytogenetic diagnostics will enable the detection of both chromosomally visible and cryptic rearrangements throughout the genome, while alleviating the need to develop FISH probes for a rapidly growing list of important cytogenetic abnormalities that require interrogation.

In conclusion, we present a 25 -year-old female with newly diagnosed B-ALL harboring a cryptic KMT2A/AFF1 fusion that was unappreciable by conventional chromosome and KMT2A BAP FISH studies. While microarray analysis revealed an initial clue to this abnormality by detection of a heterozygous deletion that spanned the $5^{\prime} A F F 1$ gene region, MPseq was required to characterize the mechanism resulting in KMT2A/AFF1 fusion. This B-ALL case demonstrates the clinical utility of MPseq in the detection of clinically significant abnormalities that may be unappreciable by traditional cytogenetic methodologies.

\section{Compliance with ethical standards}

Conflict of interest JFP, SAS, IML, BAP, CSR, JCBD, JBS, TY, PTG, RPK, and LBB declare that they have no conflict of interest. GV: Algorithms described in this manuscript for mate-pair sequencing are licensed to WholeGenome LLC owned by GV.

\section{References}

1. Borowitz MJ, Chan JKC, Downing JR et al (2017) Blymphoblastic leukaemia/lymphoma with recurrent genetic abnormalities. In: Swerdlow SH, Campo E, Harris NL et al (eds) WHO classification of tumours of haematopoietic and lymphoid tissues, revised, 4th edn. IARC, Lyon, pp 203-209

2. Meyer C, Burmeister T, Groger D et al (2018) The MLL recombinome of acute leukemias in 2017. Leukemia 32:273-284

3. Mullighan CG (2012) Molecular genetics of B-precursor acute lymphoblastic leukemia. J Clin Invest 122:3407-3415

4. Iacobucci I, Mullighan CG (2017) Genetic basis of acute lymphoblastic leukemia. J Clin Oncol 35:975-983

5. Cimino G, Elia L, Mancini M, Annino L, Anaclerico B, Fazi P, Vitale A, Specchia G, di Raimondo F, Recchia A, Cuneo A, Mecucci C, Pane F, Saglio G, Foa R, Mandelli F, GIMEMA Group (2003) Clinico-biologic features and treatment outcome of adult pro-B-ALL patients enrolled in the GIMEMA 0496 study: absence of the ALL1/AF4 and of the BCR/ABL1 fusion genes correlates with a significantly better clinical outcome. Blood 102: 2014-2020

6. Cimino G, Elia L, Rapanotti MC et al (2000) A prospective study of residual-disease monitoring of the ALL1/AF4 transcript in patients with $t(4 ; 11)$ acute lymphoblastic leukemia. Blood 95:96-101

7. Keefe JG, Sukov WR, Knudson RA, Nguyen LP, Williamson C, Sinnwell JP, Ketterling RP (2010) Development of five dual-color, double-fusion fluorescence in situ hybridization assays for the detection of common MLL translocation partners. J Mol Diagn 12: 441-452

8. Peterson JF, Sukov WR, Pitel BA, Smoley SA, Pearce KE, Meyer RG, Williamson CM, Smadbeck JB, Vasmatzis G, Hoppman NL, Greipp PT, Baughn LB, Ketterling RP (2019) Acute leukemias harboring KMT2A/MLLT10 fusion: a 10-year experience from a single genomics laboratory. Genes Chromosom Cancer. https://doi. org/10.1002/gcc.22741 
9. Drucker TM, Johnson SH, Murphy SJ, Cradic KW, Therneau TM, Vasmatzis G (2014) BIMA V3: an aligner customized for mate pair library sequencing. Bioinformatics 30:1627-1629

10. Johnson SH, Smadbeck JB, Smoley SA, Gaitatzes A, Murphy SJ, Harris FR, Drucker TM, Zenka RM, Pitel BA, Rowsey RA, Hoppman NL, Aypar U, Sukov WR, Jenkins RB, Feldman AL, Kearney HM, Vasmatzis G (2018) SVAtools for junction detection of genome-wide chromosomal rearrangements by mate-pair sequencing (MPseq). Cancer Genet 221:1-18

11. Aypar U, Smoley SA, Pitel BA, Pearce KE, Zenka RM, Vasmatzis G, Johnson SH, Smadbeck JB, Peterson JF, Geiersbach KB, van Dyke DL, Thorland EC, Jenkins RB, Ketterling RP, Greipp PT, Kearney HM, Hoppman NL, Baughn LB (2019) Mate pair sequencing improves detection of genomic abnormalities in acute myeloid leukemia. Eur J Haematol 102:87-96
12. Ramchandren R, Jazaerly T, Bluth MH, Gabali AM (2018) Molecular diagnosis of hematopoietic neoplasms: 2018 update. Clin Lab Med 38:293-310

13. Taylor J, Xiao W, Abdel-Wahab O (2017) Diagnosis and classification of hematologic malignancies on the basis of genetics. Blood $130: 410-423$

Publisher's note Springer Nature remains neutral with regard to jurisdictional claims in published maps and institutional affiliations. 